\title{
Sero-positive HIV result disclosure to sexual partner in Ethiopia: a systematic review and meta-analysis
}

Fantahun Ayenew Mekonnen ${ }^{*}$ (D), Ayenew Molla Lakew, Kindie Fentahun Muchie and Destaw Fetene Teshome

\begin{abstract}
Background: The infection of HIV continues to be an important public health problem in Ethiopia. Disclosing own HIV positive result is crucial, and considered as a good indicator of behavior change towards HIV/AIDs. A systematic review and meta-analysis was conducted to pool the prevalence of positive HIV status disclosure to sexual partners and determine the influence of selected factors.

Methods: This systematic review and meta-analysis was conducted in Ethiopia among HIV positive people receiving health care at health facilities. In this review, primary studies were searched in Medline via PubMed, Google scholar and Google up to November, 2018. Data on disclosure of HIV positive result, knowledge of partner's HIV status and prior discussion on HIV were extracted, and effect sizes like proportion and odds ratios were pooled. Heterogeneity and publication bias were assessed by chi-square and I2, and Egger test, respectively.

Results: A total of 12 studies with 4528 participants were included in to this review to estimate the prevalence of disclosure of HIV positive result to sexual partner, and 10 and 7 studies were included to determine the associations of the outcome variable with knowledge of sexual partner's HIV status and with prior discussion on HIV, respectively. The pooled prevalence of HIV status disclosure to sexual partner was 73\% (95\% Cl: 64, 82\%). Having the knowledge of sexual partner's HIV status [OR: 95\%Cl; $17.63(7.88,39.45)]$, and previous discussion on HIV [OR: $95 \% \mathrm{Cl} ; 9.24(5.56,15.37)]$ increased the disclosure of own HIV positive result to sexual partner. The sub-group analysis indicated a prevalence of $74 \%$ in Oromia, $86 \%$ in Southern Nations Nationalities and Peoples (SNNPR), $87 \%$ in Amhara, 73\% in Addis Ababa, and 54\% in Tigray.

Conclusions: Disclosure of HIV status to sexual partner is lower than expected. Knowledge of partner's HIV status and previous discussion on HIV were strong predictors of HIV positive status disclosure. Strategies helpful for encouraging open HIV discussion need to be strengthened to increase HIV positive result disclosure. Furthermore, since the heterogeneity of studies is high, large nationally representative study is suggested.
\end{abstract}

Keywords: HIV disclosure, Factors, HIV patients, systematic review, Meta-analysis

\section{Background}

HIV and AIDS still continue to be a serious global public health problem. It causes 1.8 million new infections each year. About 36.7 million people reported to live with HIV and one million people dead from HIV related illnesses in 2016. In the same year, 19.4 million people were reported to live with HIV in Eastern and Southern

\footnotetext{
* Correspondence: fantahunaye@gmail.com

Department of Epidemiology and Biostatistics, Institute of Public Health, College of Medicine and Health Sciences, University of Gondar, P.O.BOX: 96 Gondar, Ethiopia
}

(c) The Author(s). 2019 Open Access This article is distributed under the terms of the Creative Commons Attribution 4.0 International License (http://creativecommons.org/licenses/by/4.0/), which permits unrestricted use, distribution, and reproduction in any medium, provided you give appropriate credit to the original author(s) and the source, provide a link to the Creative Commons license, and indicate if changes were made. The Creative Commons Public Domain Dedication waiver (http://creativecommons.org/publicdomain/zero/1.0/) applies to the data made available in this article, unless otherwise stated. to occur, accounting 43\% of global new HIV infections, and 420,000 dead due to HIV related illnesses [1]. In Ethiopia, the problem seems to be stable though it is not as to the national and WHO target [2-4]. According to the Ethiopian Public Health Institute (EPHI) report, it was estimated that 722,248 population live with HIV, 22, 827 people develop new infections and 14,872 people die of AIDS in Ethiopia [5].

Several factors are responsible for contracting the infection, which can be grouped as behavioral, socio 
economic and demographic factors [6-8]. Individual's behavior is the most important factor for one's chance of acquiring the infection $[9,10]$. Early detection of HIV infection and disclosure of positive HIV test result, specifically, are necessary for sustainable control of HIV transmission [11-14].

The government of Ethiopia had been working to enable all people tested for HIV disclose their test results to their sexual partners regardless of the status of their tests [15]. Nevertheless, literature shows the prevalence of disclosure of HIV positive status is not only at its lower level but also highly variable across different parts of Ethiopia, as low as $41.8 \%$ [16] and as high as $93.1 \%$ [17]. Nevertheless, there was no an attempt to compile those evidences together to show overall status of the country in the disclosure of HIV positive result to sexual partner and the common factors contributing to it. Therefore, this systematic review and meta-analysis was conducted to summarize all available evidences of HIV positive result disclosure prevalence and key contributing behavioral characteristics. The finding will be helpful for the efforts that the country is making to develop efficient HIV prevention and control policies and strategies. Various data bases were explored to ensure if there were attempts to compile evidences of HIV status disclosure prevalence and associated factors in Ethiopia.

\section{Methods}

Preferred Reporting Items for Systematic Reviews and Meta-Analyses (PRISMA) checklist and flow diagram were used for designing and reporting the procedure [18]. The protocol of this systematic review and metaanalysis was registered in the Prospero database: (PROSPERO 2017: CRD42017075884).

\section{Search strategy}

A comprehensive search was carried out in Medline via PubMed, Google Scholar and Google up to November 2018. Prevalence of disclosure of HIV positive result for sexual partner and at least two associated factors were the focus of the search. The terms and/or phrases used in the search were "HIV", "Human Immune Virus", "STI", "Sexually Transmitted Diseases", "Sexually Transmitted Infection", "Disclos", "expos*", "HIV patients", "ART users", "Sexual partner", "Spouse" and "Ethiopia". There was no time limitation in the search. The search algorism was constructed using "AND" and "OR" Boolean operators.

\section{Eligibility criteria}

We included cross sectional studies that assessed prevalence of HIV positive status disclosure to sexual partner among patients in Ethiopia, restricted to English language publications, regardless of year of publication, setting, and whether they were on ART treatment or not. Disclosure of HIV status to sexual partner was the outcome variable of this review. Knowledge of partner's HIV status, and history of discussion on HIV related issues were the factors considered, in addition.

\section{Selection of studies}

Two reviewers (FAM and AML) conducted literature search independently. The two reviewers were blinded to the articles' author names, journal names and results while performing the study selection procedure. Any discrepancy between the two reviewers was resolved by negotiation or with the guidance of a third person (KFM), otherwise.

\section{Methodological quality assessment and data extraction}

After the studies were selected, critical appraisal of the included studies was carried out using the Joanna Briggs Institute Critical Appraisal Checklist for Analytical Cross Sectional Studies [19]. The items used to appraise the selected studies were: 1) explicit inclusion criteria; 2) description of study subject and setting; 3) valid and reliable measurement of exposure; 4) objective and standard criteria used; 5) identification of confounder; 6) strategies to handle confounder; 7) outcome measurement; and 8) appropriate statistical analysis. Studies scored $50 \%$ and above of the quality scale were considered low risk. The quality assessment of the studies was carried out independently by two reviewers (FAM and AML). Any disagreement between the two reviewers was resolved by the involvement of the third reviewer (DFT). Then after, data were extracted on review variables like year of publication, study setting, study location, study design, sample size, number of HIV patients who exposed their results to their sexual partners, number of patients who reported to know their sexual partners' HIV test results among those who exposed their HIV test results to their sexual partners and who did not expose, and the number of patients who reported to discuss on HIV with their sexual partners among patients who exposed their HIV test result to their sexual partner and who did not expose were extracted using the Joanna Briggs Institute (JBI) data extraction form for Prevalence and Incidence studies [20], and were entered in to a predesigned Microsoft excel sheet. The reviewers (FAM, KFM and DFT) performed the data extraction.

\section{Statistical analysis}

We examined statistical heterogeneity using the chisquare $\left(x^{2}\right)$ test. A $p$-value less than 0.05 was considered as indicative of heterogeneity. The $\mathrm{I}^{2}$ with its $\mathrm{p}$-value was computed, and the cut of values 25, 50 and $75 \%$, were used to declare the degree of heterogeneity as low, moderate and high, respectively [21]. Sensitivity and sub 
group analyses were carried out to examine if there are primary studies and study characteristics responsible for the observed heterogeneity. The Egger test was performed to assess the publication bias [22]. For the Egger test, a $p$-value less than 0.1 was assumed as a statistically significant-evidence of publication bias present. Fixed and random effects models were considered in the meta-analysis to estimate pooled prevalence and odds ratios with 95\% confidence intervals (CI) [23]. Since there was an evidence of heterogeneity, inverse variance random effects model was used as the final model of the meta-analysis. Subgroup analysis was conducted to report gender and administrative area specific effect sizes. Gender of participants (women only, and both women and men), and regions and town administrations of Ethiopia (Amhara, Oromia, SNNPR, Addis Ababa and Tigray) were considered in the sub group analysis. The Stata version 14.0 software was used for the data analysis.

\section{Results}

Study characteristics

Initially, a total of 196 studies were retrieved up to November, 2018 using the search strategies. Of these, 93 were duplicates and excluded. We also excluded 90 studies after screening their title and abstract. Full text was not found for one study and excluded. Finally, 12 articles with the full text were included in the metaanalysis to estimate the prevalence of HIV positive result disclosure to sexual partners [16, 17, 24-33] (Fig. 1). Out of these studies, 10 were included in the analysis to determine the association between knowledge of partner's HIV status with disclosure of HIV status to sexual partner [16, 17, 24-26, 29-33], and 7 in the association between history of discussion on HIV with disclosure of HIV status to sexual partner [16, 17, 24, 27, 29, 31, 32]. All the 12 studies included a total of 4528 HIV patients, whereas, the 10 and 7 studies included 3815 and 2530 patients, respectively. All the included studies were
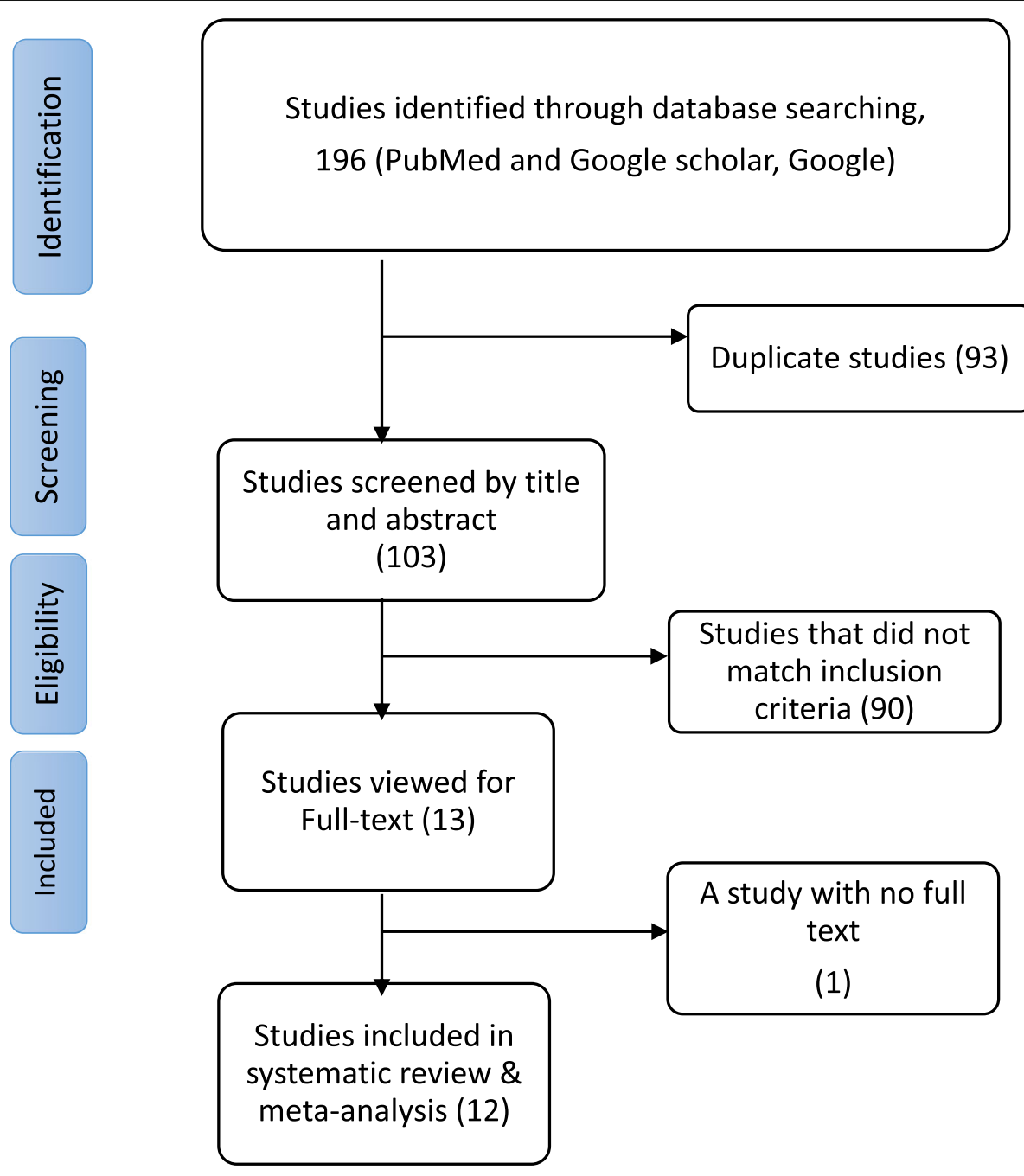

Fig. 1 PRISMA flow diagram showing identification and inclusion of studies 
conducted on four regions and one city administration of Ethiopia: four studies were from Oromia, three studies from Amhara, one study from SNNPR, one study from Addis Ababa and three studies from Tigray. Four studies included women participants only whereas 8 studies included both women and men participants. Of the 12 studies, 8 were conducted on ART patients, 2 on ART and pre ART patients and 2 on ANC attendants. Table 1 illustrates the characteristics of included studies.

\section{Quality assessment}

The included studies were assessed for quality using the JBI critical appraisal checklist for analytical cross sectional studies, and the assessment indicated that none of the included studies were of poor in quality (Table 1).

\section{Prevalence of disclosure of HIV positive status for sexual partner}

The overall prevalence of Disclosure of HIV positive status to sexual partner was found to be $73 \%$ (95\%CI: 64 , $82 \%)$, with a high level of heterogeneity $\left(p<0.001 ; \mathrm{I}^{2}=\right.$ 98.5\%) (Fig. 2). The result of the Egger test for the prevalence of disclosure of HIV positive result for sexual partners was statistically significant $(p<0.05)$, indicating the presence of evidence of publication bias.

\section{Factors associated with the disclosure of HIV positive result to sexual partners Knowledge of sexual partner's HIV status}

This meta- analysis shows knowledge of HIV patients about their sexual partners' HIV status strongly associated with the patients' disclosing of own HIV status to sexual partners. Almost the same degree and direction of association was observed at sub-population level, women only or both women and men together. Patients with the awareness of their partners' HIV status were 17.63 times more likely to expose their positive test result to their sexual partners [OR: $95 \% \mathrm{CI} ; 17.63$ (7.88, $39.45)$ ], with substantial heterogeneity $\left(p<0.001 ; \mathrm{I}^{2}=\right.$ $89.8 \%)$. When women and men together were analyzed, significant proportion of the patients who knew their partners' HIV status disclosed their HIV status to their partners as compared to those patients who did not know their partners' status [OR:95\%CI; 14.82 (5.09, 43.12)], with a significant level of heterogeneity $(\mathrm{p}<$ $\left.0.001 ; \mathrm{I}^{2}=92.4 \%\right)$. When women only was considered in the analysis, significantly more women who knew their partners' HIV status disclosed their HIV status to their sexual partner compared to those women who did not know their partners' HIV status [OR:95\%CI; 25.25 $(15.01,42.47)]$, with a low level of heterogeneity $(p<$ $0.67 ; \mathrm{I}^{2}=0.0$ ) (Fig. 3).

\section{History of discussion on HIV}

This meta-analysis revealed that patients' history of prior discussion on HIV statistically significantly associated with the disclosure of own HIV status to sexual partners. The association was almost similar in magnitude and direction at the sub-population level (women only or both women and men together). Patients with history of prior discussion on HIV were 9.24 times more likely to disclose their HIV status to their sexual partners compared to those patients who had no prior discussion on HIV [OR: 95\%CI; 9.24 $(5.56,15.37)]$, with moderate level of heterogeneity $\left(p<0.04 ; \mathrm{I}^{2}=55.5 \%\right)$ (Fig. 4$)$.

\section{Subgroup analysis}

We conducted subgroup analysis to see if the location of the studies (region), the type of participants (women only and both women and men together) were sources of the observed heterogeneity. The sub-group analysis indicated a prevalence of HIV status disclosure of $74 \%$ [(95\%CI: $57,90 \%)$ in Oromia region, $86 \%$ (95\%CI: 82, 89\%) in Southern Nations Nationalities and Peoples

Table 1 Characteristics and quality of included studies

\begin{tabular}{|c|c|c|c|c|c|c|}
\hline Author/year & Study area & Study setting & Gender & Sample size & Prevalence of HIV status disclosure & Quality \\
\hline Deribe K. et al./2008 [24] & Oromia & ART \& Pre ART & Women \&Men & 705 & 90.8 & Low risk \\
\hline Gari T. et al./2010 [25] & SNNPR & ART & Women only & 384 & 85.7 & Low risk \\
\hline Seid M. et al./2012 [17] & Amhara & ART & Women \& men & 360 & 93.1 & Low risk \\
\hline Erku T. et al./2012 [26] & Amhara & ART & Women \& men & 334 & 76.7 & Low risk \\
\hline Sendo E. et al./2013 [27] & Addis Ababa & ANC attendants & Women only & 107 & 73.0 & Low risk \\
\hline Reda A. et al./2013 [28] & Oromia & ART & Women \& men & 606 & 66.3 & Low risk \\
\hline Alemayehu M. et al./ 2014 [29] & Tigrai & ART & Women only & 315 & 64.0 & Low risk \\
\hline Alemayehu D. et al./2014 [30] & Amhara & ANC attendant & Women only & 263 & 89.7 & Low risk \\
\hline Genet M. et al./2015 [31] & Tigrai & ART & Women \& men & 324 & 57.4 & Low risk \\
\hline Alema HB..et al./2015 [16] & Tigrai & ART \& pre ART & Women \& men & 361 & 41.8 & Low risk \\
\hline Natae S. et al./2016 [32] & Oromia & ART & Women \& men & 358 & 84.9 & Low risk \\
\hline Geremew T et al./2018 [33] & Oromia & ART & Women \& men & 411 & 52.6 & Low risk \\
\hline
\end{tabular}




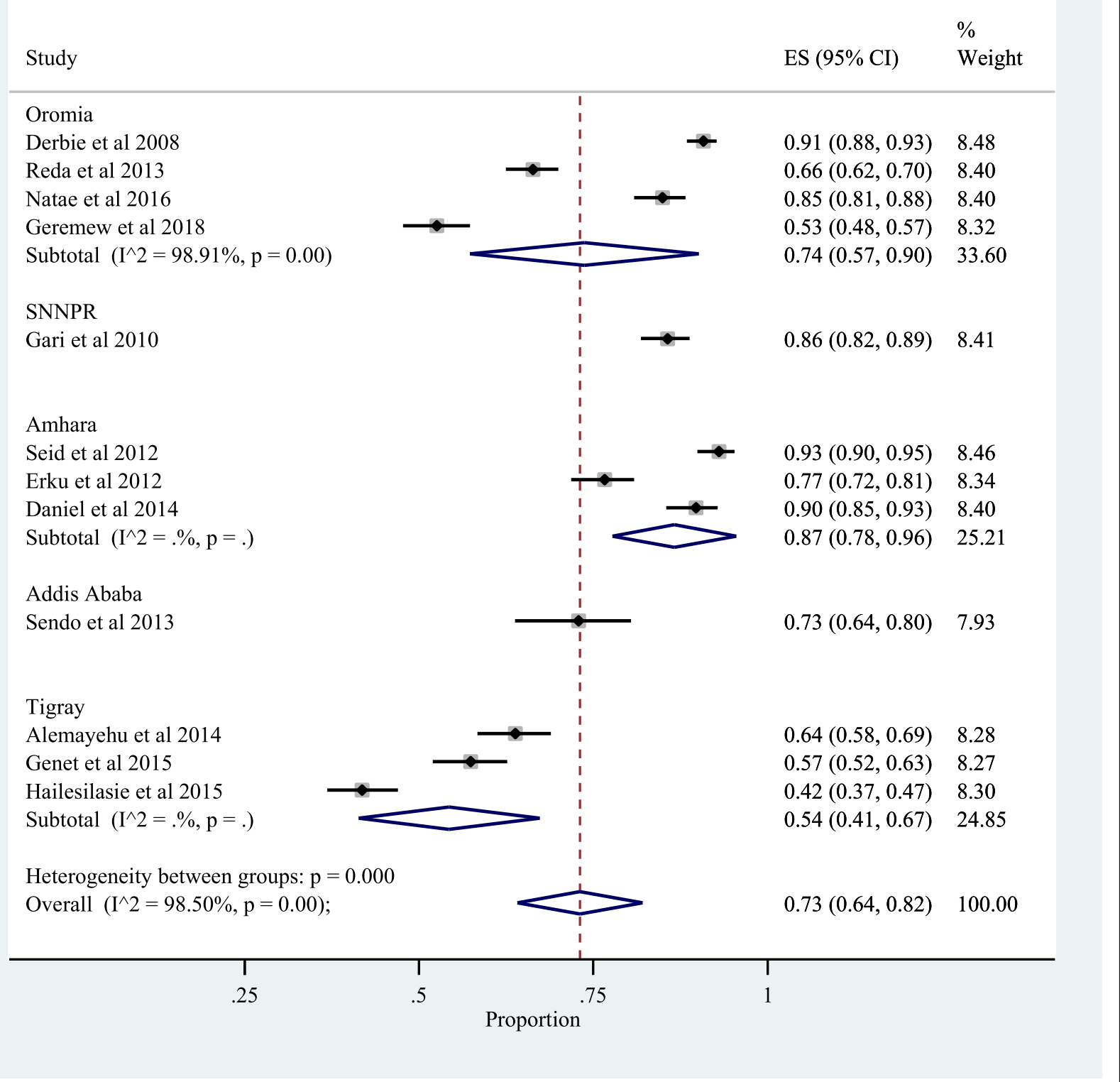

Fig. 2 Prevalence of disclosure of HIV status to sexual partner

Region (SNNPR), 87\% (95\%CI: 78, 96\%) in Amhara region, $73 \%$ (95\%CI: 64, 80\%) in Addis Ababa, and 54\% (95\%CI: 41, 67\%) in Tigray region (Fig. 2). The heterogeneity is evident among studies conducted in Oromia region only.

\section{Discussion}

The findings of this meta-analysis revealed that the prevalence of disclosure of HIV positive result to sexual partner was $73 \%$ (95\%CI: 64, 82\%). The sub-group analysis by region also showed the prevalence to be $74 \%$ (95\%CI: $57,90 \%)$ in Oromia, 86\% (95\%CI: 82, 89\%) in Southern Nations Nationalities and Peoples Region
(SNNPR), $87 \%$ (95\%CI: 78, 96\%) in Amhara, 73\% (95\%CI: 64, 80\%) in Addis Ababa, and 54\% (95\%CI: 41, $67 \%$ ) in Tigray region.

The overall HIV status disclosure prevalence in this review is similar with what was reported in Guatemala (65.6\%) [34], but lower than the finding of a study in London, England (86\%) [35]. In the contrary, this finding is higher than a study finding in El Salvador (59.7\%) [34]. It is also higher than the results of studies conducted in South Africa that 59\% of pregnant women disclosed their HIV positive results to their sexual partners [36], and in the community of rural China where $61 \%$ of participants disclosed their positive results to their 


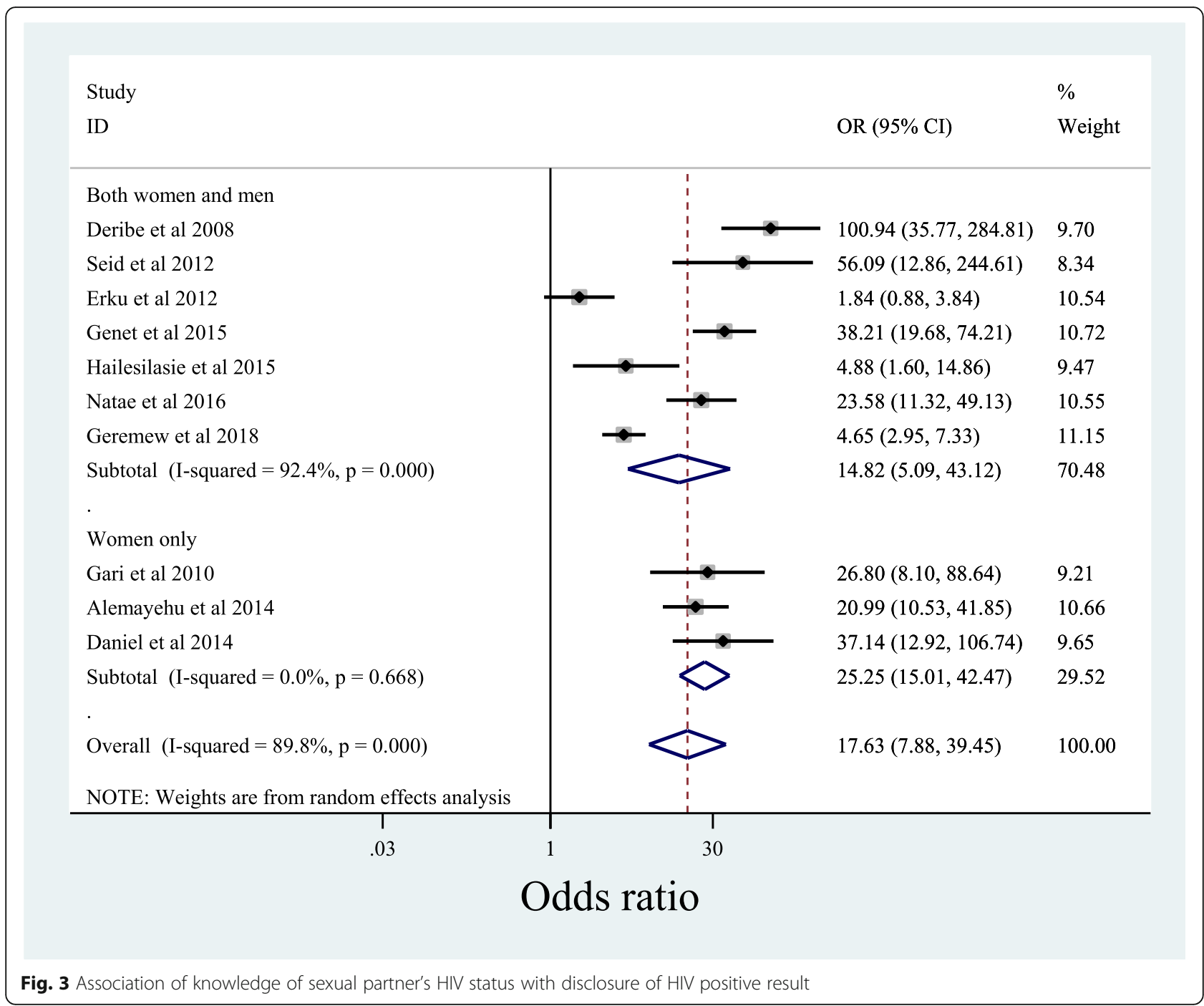

sexual partners [37]. In another study conducted among African-American women to assess self-disclosure of HIV infection to significant others, $56 \%$ of them said to have reported disclosing to their sexual partners [38], which is also lower compared to the present finding. This finding is even much higher than the findings in Nicaragua (46.2\%), Costa Rica (30.9\%), Panama (53.0\%) and Belize (28.9\%) [34]. A number of factors can be mentioned for the difference in the prevalence of disclosure of HIV positive results to sexual partners between the finding of this review and the previous studies findings. For instance, there was evidence of difference in investment for the prevention of HIV infection between Ethiopia and Central American countries that those countries has been allocating less proportion of their economy for the prevention of the infection [39]. Other possible reasons for the differences between the current and previous studies findings might be related to the design, the scope and the study participant. To be specific, the previous studies were conducted on limited contexts like either in urban or rural; ART, Pre ART or ANC patients. For example, the study in England was conducted in the urban setting, in London only, which is believed to find a relatively higher magnitude of prevalence compared to rural community. Above all, the socio -economic status and demographic characteristics differences present between our study setting and others' might be responsible for differences in the prevalence of disclosure of HIV positive test result.

Our study meta-analysis has also identified a strong association between own HIV status disclosure and knowledge of partner's HIV status, and prior discussion on HIV. Study participants who knew their partners' HIV status were more than 17.63 times more likely to disclose their HIV positive results to the sexual partners compared to those who did not have the knowledge [OR: 95\%CI; $17.63(7.88,39.45)]$. This finding coincides with a study data in London that knowledge of partner's 
Study

ID
$\%$

Weight

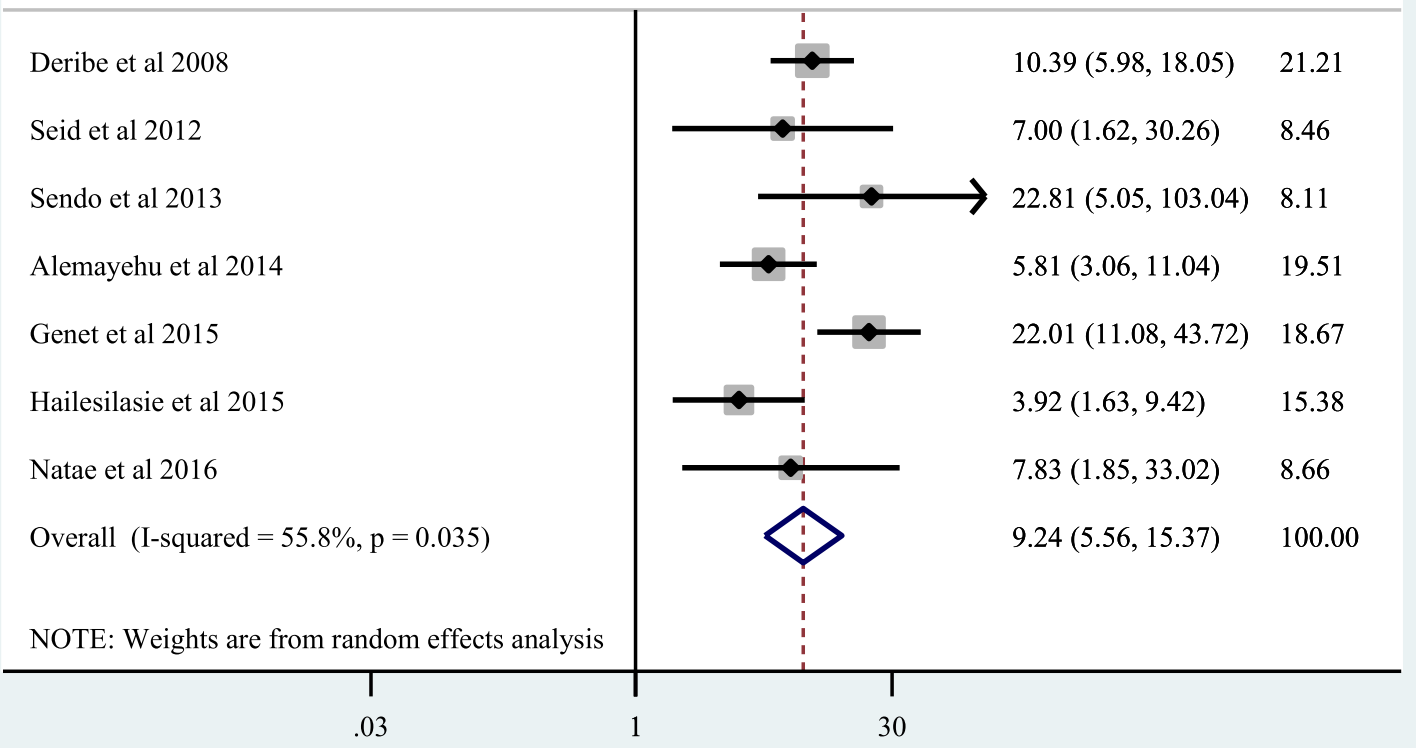

\section{Odds ratio}

Fig. 4 Association of history of discussion on HIV with disclosure of HIV positive result to sexual partner

HIV status was the only variable significantly associated with the patients likelihood of disclosing their HIV status to their partner [35]. Coming to prior discussion on HIV, the current meta-analysis revealed that study participants who had discussion on HIV previously were 9.24 times more likely to disclose their HIV positive results to their sexual partners compared to those who had not such a previous discussion [OR: 95\%CI; 9.24 $(5.56,15.37)]$. This is similar with a study finding in South Africa among pregnant women that women who had discussion on HIV testing had 4 times higher chance of disclosing their positive results to sexual partners compared to those who had not have [36].

Exhibiting both of the aforementioned factors would influence behavior towards exposing own status to sexual partners, or to significant others in general, in a number of ways. The common facilitators for disclosing through which knowledge of sexual partners' HIV status and prior discussion on HIV can work, according to several studies are; trust in the recipient of disclosure; positive experiences with previous disclosure; existence of strong social support; to gain social support; to obtain stress relief form withholding a secret; obligation and duty to inform; self-acceptance of HIV positive identity [40-42].

The authors recognized that this systematic review and meta- analysis finding might have limitations in representing the whole picture of the countries' HIV test result disclosure prevalence since almost all of the primary studies included in the meta-analysis were not evenly distributed across the five regions of Ethiopia. Even, there were no studies conducted in some regions at all. The finding need to be interpreted cautiously as the discussion was made against primary studies due to limited previous review studies. However, there was an attempt to compare the present finding against studies considered to be large studies.

\section{Conclusion}

Overall, the level of disclosure of HIV positive result is below what the government of Ethiopia intends to have in the country though there was much more investment to enable all patients regardless of their test results be empowered and inform their test results to their sexual partners. Therefore, the government need to strengthen the strategies helpful in advancing the behavior of the 
community towards open discussion on HIV testing and hence exposing own HIV test result. There need also be research aiming at determining the prevalence HIV positive test result disclosure after a specified time of knowing one's test result, could be at within the first month after being informed the result at the latest, for instance.

\section{Abbreviations}

AIDS: Acquired immune deficiency syndrome; ANC: Antenatal care; ART: Antiretroviral therapy; Cl: Confidence interval; EPHI: Ethiopian Public Health Institute; HIV: Human immune virus; JBI: Joanna Briggs Institute; PRISMA: Preferred Reporting Items for Systematic Reviews and MetaAnalyses; STI: Sexually transmitted infection; WHO: World Health Organization

\section{Acknowledgments}

We would like to thank all the authors who kindly provided the additional information for this review study.

\section{Authors' contributions}

FAM: Research idea generation, protocol development, literature searching, study quality assessment, data extraction, data analysis and report writing; AML: Protocol development, literature searching, study quality assessment, data extraction and report writing; KFM: Literature searching, data extraction and report writing, and DFT: Literature searching and data extraction. All authors read and approved the final manuscript.

\section{Funding}

We did not receive any funds for conducting this review study.

\section{Availability of data and materials}

All data are provided in the table presented in the text.

\section{Ethics approval and consent to participate}

Not applicable.

\section{Consent for publication}

Not applicable.

\section{Competing interests}

The authors declare that they have no competing interests.

Received: 5 March 2019 Accepted: 17 December 2019

Published online: 27 December 2019

\section{References}

1. UNAIDS. Fact sheet: World AIDS day 2017. Global HIV statistics, 2017

2. UNAIDS. Global AIDS response progress reporting, 2014.

3. EPRDF. Growth and transformation plan II (GTP II) (2015/16-2019/20). Addis Ababa: Ethiopian People's Revolutionary Democratic Front; 2016.

4. FMOH. Health Sector Development Program IV (2010/11-2014/15). Addis Ababa: Federal Ministry of Health; 2010.

5. EPHI. HIV Related Estimates and Projections for Ethiopia. Addis Ababa: Ethiopian Public Health Institute; 2017.

6. Kalichman SC, Kelly JA, Johnson JR, Bulto M. Factors associated with risk for HIV infection among chronic mentally ill adults. Am J Psychiatry. 1994; 151(2):221-7.

7. Chmiel JS, Detels R, Kaslow RA, Van Raden M, Kingsley LA, Brookmeyer R, et al. Factors associated with prevalent human immunodeficiency virus (HIV) infection in the multicenter AIDS cohort study. Am J Epidemiol. 1987;126(4): 568-75.

8. Ungchusak K, Rehle T, Thammapornpilap P, Spiegelman D, Brinkmann U, Siraprapasiri T. Determinants of HIV infection among female commercial sex workers in northeastern Thailand: results from a longitudinal study. JAIDS Acq Immune Def Synd. 1996;12(5):500-7.

9. Auvert B, Buvé A, Ferry B, Caraël M, Morison L, Lagarde E, et al. Ecological and individual level analysis of risk factors for HIV infection in four urban populations in sub-Saharan Africa with different levels of HIV infection. Aids. 2001;15:S15-30.

10. Hargreaves JR, Bonell CP, Boler T, Boccia D, Birdthistle I, Fletcher A, et al. Systematic review exploring time trends in the association between educational attainment and risk of HIV infection in sub-Saharan Africa. Aids. 2008:22(3):403-14

11. Hecht FM, Wang L, Collier A, Little S, Markowitz M, Margolick J, et al. A multicenter observational study of the potential benefits of initiating combination antiretroviral therapy during acute HIV infection. J Infect Dis. 2006;194(6):725-33.

12. Girardi E, Sabin CA, Antonella d'Arminio Monforte M. Late diagnosis of HIV infection: epidemiological features, consequences and strategies to encourage earlier testing. JAIDS J Acq Immune Def Synd. 2007;46:S3-8.

13. King R, Katuntu D, Lifshay J, Packel L, Batamwita R, Nakayiwa S, et al. Processes and outcomes of HIV serostatus disclosure to sexual partners among people living with HIV in Uganda. AIDS Behav. 2008;12(2):232-43.

14. De Rosa CJ, Marks G. Preventive counseling of HIV-positive men and selfdisclosure of serostatus to sex partners: new opportunities for prevention. Health Psychol. 1998;17(3):224.

15. Health FMo. Guidelines for HIV Counselling and testing in Ethiopia. Addis Ababa: Federal Minstry of Health E; 2007.

16. Alema HB, Yalew WA, Beyene MB, Woldu MG. HIV positive status disclosure and associated factors among HIV positive adults in Axum health facilities, Tigray. North Ethiopia Sci J Publ Health. 2015;3(1):61-6.

17. Seid M, Wasie B, Admassu M. Disclosure of HIV positive result to a sexual partner among adult clinical service users in Kemissie district, Northeast Ethiopia. Afr J Reprod Health. 2012;16(1):97-104.

18. LA Moher D, Tetzlaff J, Altman DG, Group P. Preferred reporting items for systematic reviews and meta-analyses: the PRISMA statement. PLoS Med. 2009;6(7):e1000097.

19. JBI. Critical Appraisal Checklist for Analytical Cross Sectional Studies: Jounna Briggs Institute; 2017. http://joannabriggs.org/research/critical-appraisaltools.html

20. JBI. The JBI data extraction form: Jonna Briggs Institute; 2016. https://wiki. joannabriggs.org/display/MANUAL/Appendix+5.2\%3A+Data+extraction+ form+for+prevalence+studies

21. Higgins JPTS, Deeks JJ, Altman DG. Measuring inconsistency in metaanalyses. BMJ Br Med J. 2003;327(7414):557.

22. Egger MSG, Schneider M, Minder C. Bias in meta-analysis detected by a simple, graphical test. BMJ. 1997;315(7109):629-34.

23. DerSimonian RLN. Meta-analysis in clinical trials. Control Clin Trials. 1986;7(3): 177-88.

24. Deribe K, Woldemichael K, Wondafrash M, Haile A, Amberbir A. Disclosure experience and associated factors among HIV positive men and women clinical service users in Southwest Ethiopia. BMC Public Health. 2008;8:81.

25. Gari T, Habte D, Markos E. HIV positive status disclosure among women attending art clinic at Hawassa University referral hospital, South Ethiopia. East Afr J Public Health. 2010;7(1):87-91.

26. Erku TA, Megabiaw B, Wubshet M. Predictors of HIV status disclosure to sexual partners among people living with HIV/AIDS in Ethiopia. Pan Afr Med J. 2012:13:87.

27. Sendo EG, Cherie A, Erku TA. Disclosure experience to partner and its effect on intention to utilize prevention of mother to child transmission service among HIV positive pregnant women attending antenatal care in Addis Ababa, Ethiopia. BMC Public Health. 2013;13:765.

28. Reda AA, Biadgilign S, Deribe K, Deribew A. HIV-positive status disclosure among men and women receiving antiretroviral treatment in eastern Ethiopia. AIDS Care. 2013;25(8):956-60.

29. Alemayehu M, Aregay A, Kalayu A, Yebyo H. HIV disclosure to sexual partner and associated factors among women attending ART clinic at Mekelle hospital, Northern Ethiopia. BMC Public Health. 2014;14:746.

30. Alemayehu D, Tadesse S, Adefris M, Birhanu Z. HIV serostatus disclosure and associated factors among HIV positive pregnant women attending antenatal care services in northwest Ethiopia. Int J Infect Control. 2014;10(4):1-8.

31. Genet M, Sebsibie G, Gultie T. Disclosure of HIV seropositive status to sexual partners and its associated factors among patients attending antiretroviral treatment clinic follow up at Mekelle hospital, Ethiopia: a cross sectional study. BMC Res Notes. 2015;8:109.

32. Natae S, Negawo M. Factors affecting HIV positive status disclosure among people living with HIV in west Showa zone, Oromia, Ethiopia; 2013. Abnorm Behav Psychol. 2016;2:114.

33. Tsige Defere Geremew RAN, Esmael JK. Sero Status Disclosure to Sexual Partner and Associated Factors among Adult HIV Positive Patients in Bale Zone Hospitals, Oromia Region, Ethiopia: Institution Based Cross-Sectional Study. Open J Epidemiol. 2018;8:43-53. 
34. Vu L, Nieto-Andrade B, DiVincenzo A, Rivas J, Firestone R, Wheeler J, et al. Effectiveness of behavior change communications for reducing transmission risks among people living with HIV in 6 countries in Central America. AIDS Behav. 2015:19(7):1203-13.

35. Dave S, Stephenson J, Mercey D, Panahmand N, Jungmann E. Sexual behaviour, condom use, and disclosure of HIV status in HIV infected heterosexual individuals attending an inner London HIV clinic. Sex Transm Infect. 2006;82(2):117-9.

36. Makin JD, Forsyth BW, Visser MJ, Sikkema KJ, Neufeld S, Jeffery B. Factors affecting disclosure in south African HIV-positive pregnant women. AIDS Patient Care STDS. 2008:22(11):907-16.

37. Qiao S, Li X, Zhou Y, Shen Z, Tang Z. AIDS impact special issue 2015: interpersonal factors associated with HIV partner disclosure among HIVinfected people in China. AIDS Care. 2016;28(sup 1):37-43.

38. Armistead $\mathrm{L}$, et al. African-American women and self-disclosure of HIV infection: rates, predictors, and relationship to depressive symptomatology. AIDS Behaviour. 1999;3:195-204.

39. Bank W. Health System Innovations in Central America. Washington DC: World Bank Working Paper No. 57; 2005.

40. Arnold RE, Flannery D, Rotheram-Borus MJ. HIV disclosure among adults living with HIV. AIDS Care. 2008;20:80-92.

41. Obermeyer CMBP, Pegurri E. Facilitating HIV disclosure across diverse settings. A Rev Am J Publ Health. 2011;101:1011-23.

42. Sowell RLPK. Understanding and responding to HIV/AIDS stigma and disclosure: an international challenge for mental health nurses. Issues Ment Health Nurs. 2010;31:394-402

\section{Publisher's Note}

Springer Nature remains neutral with regard to jurisdictional claims in published maps and institutional affiliations.

Ready to submit your research? Choose BMC and benefit from:

- fast, convenient online submission

- thorough peer review by experienced researchers in your field

- rapid publication on acceptance

- support for research data, including large and complex data types

- gold Open Access which fosters wider collaboration and increased citations

- maximum visibility for your research: over $100 \mathrm{M}$ website views per year

At BMC, research is always in progress.

Learn more biomedcentral.com/submissions 